\title{
On sums and differences of two coprime $k$ th powers
}

\author{
by
}

\author{
WENGUANG ZhAi (Jinan)
}

1. Introduction. For a fixed integer $k \geq 3$, we consider the arithmetic functions

$$
\varrho_{k}^{ \pm}(n)=\sum_{n=|m|^{k} \pm|l|^{k},(m, l)=1} 1
$$

It is easy to show that

$$
\sum_{n \leq x} \varrho_{k}^{ \pm}(n)=c_{k}^{ \pm} x^{2 / k}+b_{k}^{ \pm} x^{1 /(k-1)}+O\left(x^{1 / k}\right)
$$

for some constants $c_{k}^{ \pm}$and $b_{k}^{ \pm}$. This estimate can be slightly improved, but the problem of reducing the exponent $1 / k$ is unsolved. It is therefore natural to look for sharper estimates assuming the truth of the Riemann Hypothesis $(\mathrm{RH})$.

Let $E_{k}^{ \pm}(x)$ denote the error term in (1.1) and $\theta_{k}^{ \pm}$denote the smallest $\alpha_{k}^{ \pm}$ such that

$$
E_{k}^{ \pm}(x)=O\left(x^{\alpha_{k}^{ \pm}+\varepsilon}\right)
$$

It was noticed by E. Krätzel [6] that (under RH)

$$
\theta_{k}^{ \pm} \leq \frac{1}{k}-\frac{1}{k(3 k+2)}
$$

as a special case of a theorem due to Moroz [8].

W. G. Nowak [11] proved that if RH is true then

$$
\theta_{k}^{ \pm} \leq 127 /(140 k)
$$

for $3 \leq k \leq 7$ and

$$
\theta_{k}^{ \pm} \leq \frac{1}{k}-\frac{9 q+28}{(9 q+46) k^{2}}
$$

1991 Mathematics Subject Classification: Primary 11P21.

This work is supported partly by National Natural Science Foundation of China (Grant No. 19801021) and partly by Natural Science Foundation of Shandong Province (Grant No. Q98A02110). 
for $k \geq 8$, where $q$ is a non-negative integer such that

$$
t_{q}<k \leq t_{q+1}, \quad t_{q}=\frac{2^{q+1} 74-36}{9 q+46} .
$$

W. Müller and W. G. Nowak [9] proved (under RH) that

$$
\theta_{k}^{ \pm} \leq 37 /(41 k)
$$

for $3 \leq k \leq 6$. W. G. Nowak [12] proved (under RH) that $\theta_{3}^{+} \leq 76 / 255$.

Recently, W. G. Nowak [13] proved (under RH) that

$$
\theta_{k}^{ \pm} \leq \frac{7 k+1}{k(7 k+4)}
$$

for $k \geq 3$. For $k=3$, he proved in [15] that $\theta_{3}^{+} \leq 5 / 18$. The bound $\theta_{3}^{-} \leq 5 / 18$ is also contained in the existing literature. See Nowak [16], for example.

The aim of this paper is to study this problem for $k \geq 4$. We have

THEOREM 1. If $R H$ is true, then for any exponent pair $(\kappa, \lambda)$ such that $(3+\lambda) /(4+4 \kappa)<1-1 / k$ we have

$$
\theta_{k}^{ \pm} \leq \max \left(\frac{1}{k}-\frac{1+2 \kappa-\lambda}{1+4 \kappa-\lambda} \cdot \frac{1}{k^{2}}, \frac{173}{200 k}\right) .
$$

From Theorem 1 we can get the following

Corollary. We have

$$
\begin{gathered}
\theta_{4}^{ \pm} \leq 173 / 800, \quad \theta_{5}^{ \pm} \leq 251 / 1450, \quad \theta_{6}^{ \pm} \leq 77 / 522, \\
\theta_{k}^{ \pm} \leq \frac{1}{k}-\frac{9}{13} \cdot \frac{1}{k^{2}} \quad(k \geq 7) .
\end{gathered}
$$

For $\theta_{4}^{+}$, we can get a slightly better estimate. We have

Theorem 2. If $R H$ is true, then

$$
\theta_{4}^{+} \leq 107 / 512 \text {. }
$$

The structure of the paper is as follows. In Section 2, some preliminary lemmas are quoted. In Section 3, we study the properties of the function $Z_{k}^{ \pm}(s)$. We estimate an exponential sum involving the Möbius function in Section 4. The proofs of Theorem 1 and the Corollary are given in Section 5. We prove Theorem 2 in Section 6.

Notations. $\psi(t)=\{t\}-1 / 2,\{t\}$ is the fractional part of $t . e(t)=e^{2 \pi i t}$. $\mu(n)$ denotes the Möbius function. $\varepsilon$ denotes a small positive constant which may be different at each occurrence. We use $\mathrm{SC}\left(\sum\right)$ to denote the summation conditions of the sum $\sum$ if these conditions are complicated. For example, instead of

$$
F(x)=\sum_{a \leq n \leq x} f(n)
$$


we can write

$$
F(x)=\sum f(n), \quad \operatorname{SC}\left(\sum\right): a \leq n \leq x .
$$

The author wants to thank Professor W. G. Nowak for kindly sending reprints of some of his papers.

\section{Some preliminary lemmas}

LEMma 1. Let $F(x)$ be a real differentiable function such that $F^{\prime}(x)$ is monotonic and $\left|F^{\prime}(x)\right| \geq m>0, G(x)$ is a positive monotonic function satisfying $|G(x)| \leq G$ for $a \leq x \leq b$. Then

$$
\left|\int_{a}^{b} G(x) e^{i F(x)} d x\right| \leq 4 G m^{-1}
$$

Lemma 2. Let $\mathcal{X}$ and $\mathcal{Y}$ be two finite sets of real numbers, $\mathcal{X} \subset[-X, X]$, $\mathcal{Y} \subset[-Y, Y]$. Then for any complex functions $u(x)$ and $v(y)$ we have

$$
\begin{aligned}
& \left|\sum_{x \in \mathcal{X}} \sum_{y \in \mathcal{Y}} u(x) v(y) e(x y)\right|^{2} \\
& \quad \leq 20(1+X Y) \sum_{\substack{x, x^{\prime} \in \mathcal{X} \\
\left|x-x^{\prime}\right| \leq Y^{-1}}}\left|u(x) u\left(x^{\prime}\right)\right| \sum_{\substack{y, y^{\prime} \in \mathcal{Y} \\
\left|y-y^{\prime}\right| \leq X^{-1}}}\left|v(y) v\left(y^{\prime}\right)\right| .
\end{aligned}
$$

Lemma 3. Let $\alpha, \alpha_{1}, \alpha_{2}, z$ be real numbers such that $z \alpha \alpha_{1} \alpha_{2} \neq 0, \alpha \notin \mathbb{N}$. Let $M \geq 1, M_{1} \geq 1, M_{2} \geq 1$ and let $a_{m}$ and $b_{m_{1} m_{2}}$ be complex numbers with $\left|a_{m}\right| \leq 1$ and $\left|b_{m_{1} m_{2}}\right| \leq 1$. Let $F=|z| M^{\alpha} M_{1}^{\alpha_{1}} M_{2}^{\alpha_{2}}$. If $F \geq M_{1} M_{2}$, then

$$
\begin{aligned}
& \sum_{m \sim M} \sum_{m_{1} \sim M_{1}} \sum_{m_{2} \sim M_{2}} a_{m} b_{m_{1} m_{2}} e\left(z m^{\alpha} m_{1}^{\alpha_{1}} m_{2}^{\alpha_{2}}\right) \\
& \ll M M_{1} M_{2} \log \left(2 M_{1} M_{2}\right)\left\{\left(M_{1} M_{2}\right)^{-1 / 2}\right. \\
&\left.+\left(F /\left(M_{1} M_{2}\right)\right)^{\kappa /(2(1+\kappa))} M^{-(1+\kappa-\lambda) /(2(1+\kappa))}\right\} .
\end{aligned}
$$

Lemma 4. For any $J \geq 2$, we have

$$
\psi(t)=\sum_{1 \leq|h| \leq J} a(h) e(h t)+O\left(\sum_{|h| \leq J} b(h) e(h t)\right)
$$

with

$$
a(h) \ll|h|^{-1}, \quad b(h) \ll J^{-1} .
$$

Lemma 5. For fixed $k \geq 3$, let

$$
r_{k}^{+}(n)=\sum_{n=|m|^{k}+|l|^{k}} 1, \quad R_{k}^{+}(x)=\sum_{n \leq x} r_{k}(n) .
$$


Then $R_{k}^{+}(x)=H_{k}(x)+\Delta_{k 1}^{+}(x)+\Delta_{k 2}^{+}(x)$, where

$$
\begin{aligned}
H_{k}(x)= & c_{k}^{\prime} x^{2 / k}, \quad c_{k}^{\prime}=\frac{2 \Gamma^{2}(1 / k)}{k \Gamma(2 / k)}, \\
\Delta_{k 1}^{+}(x)= & \frac{8 \Gamma(1 / k)}{k \pi} x^{1 / k-1 / k^{2}} \sum_{l=1}^{\infty} \frac{1}{k}\left(\frac{k}{2 \pi l}\right)^{1 / k} \cos 2 \pi\left(l x^{1 / k}-\frac{1}{4}\left(1+\frac{1}{k}\right)\right) \\
& +O(1), \\
\Delta_{k 2}^{+}(x)= & -8 \sum_{x / 2 \leq n^{k} \leq x} \psi\left(\left(x-n^{k}\right)^{1 / k}\right)+O(1), \\
\Delta_{k 1}^{+}(x)+ & \Delta_{k 2}^{+}(x) \ll x^{1 / k-1 / k^{2}} .
\end{aligned}
$$

LEMMA 5a. Let

$$
r_{k}^{-}(n)=\sum_{n=|m|^{k}-|l|^{k}} 1, \quad R_{k}^{-}(x)=\sum_{n \leq x} r_{k}^{-}(n) .
$$

Then $R_{k}^{-}(x)=a_{k}^{-} x^{2 / k}+b_{k}^{-} x^{1 /(k-1)}+\Delta_{k 1}^{-}(x)+\Delta_{k 2}^{-}(x)$, with

$$
\begin{aligned}
& \Delta_{k 1}^{-}(x)=c_{k}^{-} \sum_{l=1}^{\infty} l^{-1-1 / k} \sin \left(2 \pi l x^{1 / k}+\frac{\pi}{2 k}\right), \\
& \Delta_{k 2}^{-}(x)=4 \Sigma_{k 1}(x)-4 \Sigma_{k 2}(x)+O(1), \\
& \Sigma_{k 1}(x)=\sum_{x^{1 / k}<m \leq \lambda x^{1 / k}} \psi_{1}\left(\left(m^{k}-x\right)^{1 / k}\right), \\
& \Sigma_{k 2}(x)=\sum_{1<m \leq \delta x^{1 / k}} \psi_{0}\left(N_{k}(m, x)\right) .
\end{aligned}
$$

Here $\delta$ is an arbitrarily small positive constant, $\lambda=\lambda(\delta) \rightarrow \infty$ as $\delta \rightarrow 0$, and

$$
\begin{array}{ll}
\psi_{0}(v)=\psi_{1}(v)=v-[v]-1 / 2 & \text { for } v \notin \mathbb{Z}, \\
\psi_{0}(v)=\psi_{1}(v)=1 / 2 & \text { for } v \in \mathbb{Z} .
\end{array}
$$

The function $v=N_{k}(w, x)$ is defined by the equation

$$
(v+w)^{k}-v^{k}=x \quad\left(v, w, x \in \mathbb{R}^{+}, w<x^{1 / k}\right) .
$$

LEMMA 6. We have the following estimates:

$$
\begin{gathered}
\sum_{n=1}^{\infty} \frac{r_{k}^{ \pm 2}(n)}{n^{\sigma}} \ll 1, \quad \sum_{n=1}^{\infty} \frac{r_{k}^{ \pm}(n)}{n^{\sigma}} \ll 1, \quad \sigma>2 / k ; \\
\sum_{n \leq x} \frac{r_{k}^{ \pm}(n)}{n^{2 / k}} \ll \log x ; \quad \sum_{n \leq x} \frac{r_{k}^{ \pm}(n)}{n^{\sigma}} \ll x^{2 / k-\sigma}, \quad 0<\sigma<2 / k .
\end{gathered}
$$

Lemma 1 is formula (2.3) of Ivić [4]. Lemma 2 is Proposition 1 of Fouvry and Iwaniec [2]. Lemma 3 is Theorem 2 of Baker [1]. Lemma 4 can be found 
in Vaaler [17]. Lemma 5 is contained in Section 3.3 of [5]. Lemma $5 \mathrm{a}$ is formula (5) of Müller and Nowak [10]. Lemma 6 immediately follows from Lemmas 5 and 5 a by partial summation.

3. Expression of the error term. In this section we shall give an expression of $E_{k}^{ \pm}(x)$ subject to RH. Following the work of W. G. Nowak [13], we first study the functions

$$
Z_{k}^{ \pm}(s)=\sum_{n=1}^{\infty} \frac{r_{k}^{ \pm}(n)}{n^{s}}
$$

The following Lemma 7 and Lemma 7a play the key roles in our proofs, from which we can obtain better mean-value results on $Z_{k}^{ \pm}(s)$. Thus we improve Nowak's previous results on the two functions.

Lemma 7. Suppose $|t| \geq 2$ and $M \geq(10 k)^{10 k}|t|^{k}$. Then

$$
\int_{M}^{2 M} \Delta_{k 1}^{+}(x) x^{i t} d x \ll M, \quad \int_{M}^{2 M} \Delta_{k 2}^{+}(x) x^{i t} d x \ll M .
$$

Proof. We first prove the first assertion. Obviously

$$
\begin{aligned}
& \int_{M}^{2 M} \Delta_{k 1}^{+}(x) x^{i t} d x \\
= & c_{1}(k) \sum_{l=1}^{\infty} \frac{1}{l^{1+1 / k}} \int_{M}^{2 M} x^{1 / k-1 / k^{2}} \cos 2 \pi\left(l x^{1 / k}-\frac{1}{4}\left(1+\frac{1}{k}\right)\right) x^{i t} d x \\
& +O(M) \\
\ll & M+\sum_{l=1}^{\infty} \frac{1}{l^{1+1 / k}}\left|\int_{M}^{2 M} x^{1 / k-1 / k^{2}} e\left(l x^{1 / k}-\frac{1}{4}\left(1+\frac{1}{k}\right)+\frac{t \log x}{2 \pi}\right) d x\right| \\
& +\sum_{l=1}^{\infty} \frac{1}{l^{1+1 / k}}\left|\int_{M}^{2 M} x^{1 / k-1 / k^{2}} e\left(-l x^{1 / k}+\frac{1}{4}\left(1+\frac{1}{k}\right)+\frac{t \log x}{2 \pi}\right) d x\right| .
\end{aligned}
$$

Let

$f_{1}(x)=l x^{1 / k}-\frac{1}{4}\left(1+\frac{1}{k}\right)+\frac{t \log x}{2 \pi}, \quad f_{2}(x)=-l x^{1 / k}+\frac{1}{4}\left(1+\frac{1}{k}\right)+\frac{t \log x}{2 \pi}$.

Then

$$
\left|f_{1}^{\prime}(x)\right| \gg l M^{1 / k-1}, \quad\left|f_{2}^{\prime}(x)\right| \gg l M^{1 / k-1} .
$$

Hence the first assertion of Lemma 7 follows from (3.1) by Lemma 1.

Now we consider the second assertion. We write

$$
\Delta_{k 2}^{+}(x)=-8 \sum^{1} \sum^{2} \psi\left(\left(x-n^{k}\right)^{1 / k}\right)+O_{\varepsilon}(1),
$$


where

$$
\begin{aligned}
& \mathrm{SC}\left(\sum^{1}\right): 1 \leq v \leq \frac{\log \varepsilon M^{1 / k}}{\log 2}, \\
& \mathrm{SC}\left(\sum^{2}\right): x\left(1-2^{-v}\right)<n^{k} \leq x\left(1-2^{-v-1}\right),
\end{aligned}
$$

and $\varepsilon$ is a fixed small positive constant. It suffices to estimate

$$
\int_{v}=\int_{M}^{2 M} \sum^{2} \psi\left(\left(x-n^{k}\right)^{1 / k}\right) x^{i t} d x
$$

for each fixed $v$.

We take $J=M^{1 / k} 2^{-v}$ in Lemma 4 . Change the order of summation and integration and then use Lemma 5 to get

$$
\begin{aligned}
\int_{v}= & \int_{M}^{2 M} \sum^{2} \psi\left(\left(x-n^{k}\right)^{1 / k}\right) x^{i t} d x \\
= & \sum^{3} \int_{a(n)}^{b(n)} \psi\left(\left(x-n^{k}\right)^{1 / k}\right) x^{i t} d x \\
= & \sum^{3} \sum_{1 \leq|h| \leq J} \frac{1}{2 \pi i h} \int_{a(n)}^{b(n)} e\left(h\left(x-n^{k}\right)^{1 / k}\right) x^{i t} d x \\
& +O\left(\sum^{3} \sum_{1 \leq|h| \leq J} \frac{1}{J}\left|\int_{a(n)}^{b(n)} e\left(h\left(x-n^{k}\right)^{1 / k}\right) x^{i t} d x\right|\right)+O\left(M 2^{-v}\right),
\end{aligned}
$$

where

$$
\mathrm{SC}\left(\sum^{3}\right): M\left(1-2^{-v}\right)<n^{k} \leq 2 M\left(1-2^{-v-1}\right)
$$

and $[a(n), b(n)]$ is a subinterval of $[M, 2 M]$.

Let $f_{3}(x)=h\left(x-n^{k}\right)^{1 / k}+(t \log x) /(2 \pi)$. Then

$$
\left|f_{3}^{\prime}(x)\right|=\left|\frac{1}{k} h\left(x-n^{k}\right)^{1 / k-1}+\frac{t}{2 \pi x}\right| \gg h M^{1 / k-1} 2^{v(1-1 / k)} .
$$

Again by Lemma 1,

$$
\int_{v} \ll \sum^{3} \sum_{h} \frac{1}{h}\left(h M^{1 / k-1} 2^{v(1-1 / k)}\right)^{-1}+M 2^{-v} \ll M 2^{-v(1-1 / k)} .
$$

Hence the second assertion follows.

LEMMA 8. $Z_{k}^{+}(s)$ has the following properties:

(1) $Z_{k}^{+}(s)$ has an analytic continuation to $\sigma>1 / k-1 / k^{2}$ with the exception of one simple pole at $s=2 / k$. 
(2) We have

$$
Z_{k}^{+}(\sigma+i t) \ll \min \left(\log |t|, \frac{1}{\sigma-2 / k}\right), \quad \sigma \geq 2 / k,|t| \geq 2 .
$$

(3) We have

$$
Z_{k}^{+}(\sigma+i t) \ll|t|^{\left(1 / k+1 / k^{2}\right)(2 / k-\sigma)} \log |t|
$$

uniformly for $1 / k-1 / k^{2}<\sigma_{1} \leq \sigma \leq 2 / k,|t| \geq 2$.

(4) For any real parameter $T \geq 10$, we have

$$
\int_{T}^{2 T}\left|Z_{k}^{+}\left(\frac{3}{2 k}+i t\right)\right|^{2} d t \ll T \log T .
$$

Proof. Suppose $X \geq 2$ is a parameter. For $\sigma>2 / k$, by Stieltjes integration we get

$$
\begin{aligned}
Z_{k}^{+}(s)= & \sum_{n \leq X} \frac{r_{k}^{+}(n)}{n^{s}}+\int_{X}^{\infty} \omega^{-s} d R_{k}^{+}(\omega) \\
= & \sum_{n \leq X} \frac{r_{k}^{+}(n)}{n^{s}}+\int_{X}^{\infty} \omega^{-s} d\left(c_{k}^{\prime} \omega^{2 / k}+\Delta_{k 1}^{+}(\omega)+\Delta_{k 2}^{+}(\omega)\right) \\
= & \sum_{n \leq X} \frac{r_{k}^{+}(n)}{n^{s}}+\frac{2}{k} c_{k}^{\prime} \frac{X^{2 / k-s}}{s-2 / k}-X^{-s}\left(\Delta_{k 1}^{+}(X)+\Delta_{k 2}^{+}(X)\right) \\
& +s \int_{X}^{\infty} \frac{\Delta_{k 1}^{+}(\omega)+\Delta_{k 2}^{+}(\omega)}{\omega^{s+1}} d \omega .
\end{aligned}
$$

Since $\Delta_{k 1}^{+}(\omega)+\Delta_{k 2}^{+}(\omega) \ll \omega^{1 / k-1 / k^{2}}$, the above integral converges absolutely for $\sigma>1 / k-1 / k^{2}$. Hence the first assertion of Lemma 8 follows.

The second assertion follows from (3.5) and Lemma 6 .

Suppose $1 / k-1 / k^{2}<\sigma_{1}<2 / k$ is fixed. Then by Lemmas 5 and 6 we have

$$
Z_{k}^{+}\left(\sigma_{1}+i t\right) \ll X^{2 / k-\sigma_{1}}+|t| X^{1 / k-1 / k^{2}-\sigma_{1}} \ll|t|^{\left(1 / k+1 / k^{2}\right)\left(2 / k-\sigma_{1}\right)}
$$

by choosing $X=|t|^{1 / k+1 / k^{2}}$. Hence the third assertion of Lemma 8 follows from the well-known Phragmen-Lindelöf argument.

Now we prove the fourth assertion. Take $X=(10 k)^{10 k} T^{k}$. By Lemma 6 we have

$$
\int_{X}^{\infty} \frac{\Delta_{k 1}^{+}(\omega)+\Delta_{k 2}^{+}(\omega)}{\omega^{s+1}} d \omega \ll X^{-3 /(2 k)} .
$$


Inserting into (3.5) we get

$$
Z_{k}^{+}\left(\frac{3}{2 k}+i t\right)=\sum_{n \leq X} \frac{r_{k}^{+}(n)}{n^{3 /(2 k)+i t}}+O(1) .
$$

Squaring (3.8) and integrating over $T \leq t \leq 2 T$ gives

$$
\begin{aligned}
\int_{T}^{2 T}\left|Z_{k}^{+}\left(\frac{3}{2 k}+i t\right)\right|^{2} d t & \ll \int_{T}^{2 T}\left|\sum_{n \leq X} \frac{r_{k}^{+}(n)}{n^{3 /(2 k)+i t}}\right|^{2} d t+T \\
& =\sum_{m, n \leq X} \frac{r_{k}^{+}(m) r_{k}^{+}(n)}{(m n)^{3 /(2 k)}} \int_{T}^{2 T}\left(\frac{m}{n}\right)^{i t} d t+T \\
& =\sum_{m=n}+\sum_{m \neq n}+T
\end{aligned}
$$

By Lemma 6 we have

$$
\sum_{m=n} \ll T \sum_{n \leq X} \frac{r_{k}^{+2}(n)}{n^{3 / k}} \ll T .
$$

By Lemma 1,

$$
\begin{aligned}
\sum_{m \neq n} & \ll \sum_{n<m \leq X} \frac{r_{k}^{+}(m) r_{k}^{+}(n)}{(m n)^{3 /(2 k)}} \min \left(T, \frac{1}{\log (m / n)}\right) \\
& =\sum^{1}+\sum^{2}+\sum^{3}, \text { say },
\end{aligned}
$$

where

$$
\begin{aligned}
& \mathrm{SC}\left(\sum^{1}\right): n \leq X, n<m \leq n e^{1 / T}, \\
& \mathrm{SC}\left(\sum^{2}\right): n \leq X, n e^{1 / T}<m \leq 2 n, \\
& \mathrm{SC}\left(\sum^{3}\right): 2 n<m \leq X .
\end{aligned}
$$

By Lemmas 5 and 6 we have

$$
\begin{aligned}
\sum^{1} & \ll T \sum_{n \leq X} \frac{r_{k}^{+}(n)}{n^{3 / k}} \sum_{n<m \leq n e^{1 / T}} r_{k}^{+}(m) \\
& \ll T \sum_{n \leq X} \frac{r_{k}^{+}(n)}{n^{3 / k}}\left(\left(e^{1 / T}-1\right) n^{2 / k}+O\left(n^{1 / k-1 / k^{2}}\right)\right) \\
& \ll T\left(e^{1 / T}-1\right) \sum_{n \leq X} \frac{r_{k}^{+}(n)}{n^{1 / k}}+T \sum_{n \leq X} \frac{r_{k}^{+}(n)}{n^{2 / k+1 / k^{2}}} \\
& \ll X^{1 / k}+T \ll T
\end{aligned}
$$


and

$$
\sum^{3} \ll \sum_{n \leq X} \frac{r_{k}^{+}(n)}{n^{3 /(2 k)}} \sum_{m \leq X} \frac{r_{k}^{+}(m)}{m^{3 /(2 k)}} \ll X^{1 /(2 k)} X^{1 /(2 k)} \ll T .
$$

It remains to estimate $\sum^{2}$. Let $m=n+r$ and notice

$$
\frac{1}{\log (m / n)}=\frac{1}{\log (1+r / n)} \ll \frac{n}{r}
$$

we get

$$
\sum^{2} \ll \sum_{n \leq X} \frac{r_{k}^{+}(n)}{n^{3 / k-1}} \sum^{4} \frac{r_{k}^{+}(n+r)}{r},
$$

where

$$
\mathrm{SC}\left(\sum^{4}\right): \max \left(1, n\left(e^{1 / T}-1\right)\right) \leq r \leq n .
$$

Using a splitting argument and then using Lemma 5 gives

$$
\begin{aligned}
\sum^{4} & \ll \log n \cdot \max _{a \ll n} \sum_{a<r \leq 2 a} \frac{r_{k}^{+}(n+r)}{r} \\
& \ll \log n \cdot \max _{a \ll n} a^{-1} \sum_{a<r \leq 2 a} r_{k}^{+}(n+r) \\
& \ll \log n \cdot \max _{a \ll n} a^{-1} \sum_{n+a<r \leq n+2 a} r_{k}^{+}(r) \\
& \ll \log n \cdot \max _{a \ll n} a^{-1}\left((n+2 a)^{2 / k}-(n+a)^{2 / k}+O\left(n^{1 / k-1 / k^{2}}\right)\right) \\
& \ll \log n \cdot \max _{a \ll n}\left(n^{2 / k-1}+n^{1 / k-1 / k^{2}} a^{-1}\right) \\
& \ll n^{2 / k-1} \log n+T n^{1 / k-1 / k^{2}-1} \log n,
\end{aligned}
$$

where in the last step we used the fact that $r \gg n\left(e^{1 / T}-1\right) \gg n / T$.

Inserting (3.15) into (3.14) we get

$$
\begin{aligned}
\sum^{2} & \ll \sum_{n \leq X} \frac{r_{k}^{+}(n)}{n^{3 / k-1}}\left(n^{2 / k-1} \log n+T n^{1 / k-1 / k^{2}-1} \log n\right) \\
& \ll X^{1 / k} \log X+T \log T \ll T \log T .
\end{aligned}
$$

Now the fourth assertion of Lemma 8 follows from (3.9) to (3.16).

Lemma 7a. Suppose $|t| \geq 2, c(k, \delta)$ is a sufficiently large constant and $M \geq c(k, \delta)|t|^{k}$. Then

$$
\int_{M}^{2 M} \Delta_{k 1}^{-}(x) x^{i t} d x \ll M, \quad \int_{M}^{2 M} \Delta_{k 2}^{-}(x) x^{i t} d x \ll M .
$$


Proof. The first assertion is actually the first assertion of Lemma 7. To prove the second assertion we only need to show that

$$
\int_{M}^{2 M} \Sigma_{k 1}(x) x^{i t} d x \ll M
$$

and

$$
\int_{M}^{2 M} \Sigma_{k 2}(x) x^{i t} d x \ll M .
$$

The proof of (3.17) is similar to that of the second assertion of Lemma 7.

Similar to the proof of Lemma 7, we change the order of integration and summation, and then use Lemma 1 after appealing to Lemma 4; and then (3.18) follows if $c(k, \delta)$ is sufficiently large.

LEMMA 8 a. $Z_{k}^{-}(s)$ has the following properties:

(1) $Z_{k}^{-}(s)$ has an analytic continuation to $\sigma>1 / k-1 / k^{2}$ with the exception of two simple poles at $s=2 / k$ and $s=1 /(k-1)$.

(2) We have

$$
Z_{k}^{-}(\sigma+i t) \ll \min \left(\log |t|, \frac{1}{\sigma-2 / k}\right), \quad \sigma \geq 2 / k,|t| \geq 2 .
$$

(3) We have

$$
Z_{k}^{-}(\sigma+i t) \ll|t|^{\left(1 / k+1 / k^{2}\right)(2 / k-\sigma)} \log |t|
$$

uniformly for $1 / k-1 / k^{2}<\sigma_{1} \leq \sigma \leq 2 / k,|t| \geq 2$.

(4) For any real parameter $T \geq 10$, we have

$$
\int_{T}^{2 T}\left|Z_{k}^{-}\left(\frac{3}{2 k}+i t\right)\right|^{2} d t \ll T \log T .
$$

Proof. This lemma can be proved in the same way as Lemma 8.

In the same way as in Nowak [13], we can get the following

Proposition 1. If RH is true, $10<y<x^{1 / k}$, then

$$
E_{k}^{ \pm}(x)=\sum_{d \leq y} \mu(d)\left(\Delta_{k 1}^{ \pm}\left(\frac{x}{d^{k}}\right)+\Delta_{k 2}^{ \pm}\left(\frac{x}{d^{k}}\right)\right)+O\left(x^{3 /(2 k)+\varepsilon} y^{-1}\right) .
$$

4. On an exponential sum involving the Möbius function. In this section we shall estimate the exponential sum

$$
S(W, D)=\sum_{d \sim D} \mu(d) e(W / d)
$$

where $W$ and $D \geq 5$ are two positive numbers with $D \ll W^{1-\varepsilon}$. 
Lemma 9. Suppose $a_{m} \ll 1$ is any complex number, $0<\alpha<1 / 2$ is a fixed real number. If $M \ll D^{\alpha}$ and $D \ll M N \ll D$, then for any exponent pair $(\kappa, \lambda)$ we have

$$
S_{I}=\sum_{m \sim M} a_{m} \sum_{n \sim N} e\left(\frac{W}{m n}\right) \ll \frac{D^{2}}{W}+W^{\kappa /(2(1+\kappa))} D^{(3+\lambda) /(4(1+\kappa))} .
$$

Proof. This estimate easily follows from using the exponent pair $(\kappa /(2(1+\kappa)), 1 / 2+\lambda /(2(1+\kappa)))$ directly to the sum over $n$ and noticing $\alpha<1 / 2$.

Lemma 10. Suppose $a_{m} \ll 1$ and $b_{n} \ll 1$ are any complex numbers, $0<\alpha<1 / 2$ is a fixed real number. If $D^{\alpha} \ll N \ll D^{1 / 2}$ and $M N \sim D$, then for any exponent pair $(\kappa, \lambda)$ we have

$$
\begin{aligned}
S_{I I} & =\sum_{m \sim M} a_{m} \sum_{n \sim N} b(n) e\left(\frac{W}{m n}\right) \\
& \ll\left(W^{\kappa /(2(1+\kappa))} D^{(3+\lambda) /(4(1+\kappa))}+D^{1-\alpha / 2}+D^{3 / 2} W^{-1 / 2}\right) \log ^{2} D .
\end{aligned}
$$

Proof. Let $F=W / D$. If $F<N$, then using Lemma 2 we get

$$
S_{I I} \ll M N F^{-1 / 2} \ll D^{3 / 2} W^{-1 / 2} .
$$

If $F \geq N$, by Lemma 3 we get (take $m_{1}=1, m_{2}=n$ )

$$
\begin{aligned}
S_{I I} \log ^{-1} D & \ll M N\left(N^{-1 / 2}+(F / N)^{\kappa /(2(1+\kappa))} M^{-(1+\kappa-\lambda) /(2(1+\kappa))}\right) \\
& \ll D^{1-\alpha / 2}+F^{\kappa /(2(1+\kappa))} N^{(2+\kappa) /(2(1+\kappa))} M^{(1+\lambda+\kappa) /(2(1+\kappa))} \\
& \ll W^{\kappa /(2(1+\kappa))} D^{(3+\lambda) /(4(1+\kappa))}+D^{1-\alpha / 2},
\end{aligned}
$$

where we used the fact that $D^{\alpha} \ll N \ll D^{1 / 2}$.

Now we prove the following

Proposition 2. Suppose $0<\alpha<1 / 2$ is fixed. Then for any exponent pair $(\kappa, \lambda)$ we have

$$
D^{-\varepsilon} S(W, D) \ll W^{\kappa /(2(1+\kappa))} D^{(3+\lambda) /(4(1+\kappa))}+D^{1-\alpha / 2}+D^{3 / 2} W^{-1 / 2} .
$$

Pro of. We use the skillful decomposition due to Montgomery and Vaughan [7] and write

$$
S(W, D)=\Sigma_{1}+\Sigma_{2}+\Sigma_{3}, \quad \text { say }
$$

where

$$
\begin{aligned}
& \Sigma_{1}=-\sum_{m \leq U} \xi_{m} \sum_{D / m<n \leq D^{\prime} / m} e\left(\frac{W}{m n}\right), \\
& \Sigma_{2}=-\sum_{U<m \leq U^{2}} \xi_{m} \sum_{D / m<n \leq D^{\prime} / m} e\left(\frac{W}{m n}\right),
\end{aligned}
$$




$$
\begin{gathered}
\xi_{m}=\sum_{m=d_{1} d_{2}, d_{1}, d_{2} \leq U} \mu\left(d_{1}\right) \mu\left(d_{2}\right) \ll m^{\varepsilon}, \\
\Sigma_{3}=-\sum_{m>U, n>U, D<m n<D^{\prime}} \mu(m) \eta_{n} e\left(\frac{W}{m n}\right), \quad \eta_{n}=\sum_{d \mid n, d \leq U} \mu(d) \ll n^{\varepsilon} .
\end{gathered}
$$

We choose $U=D^{\alpha}$. Use Lemma 9 to estimate $\Sigma_{1}$ and Lemma 10 to estimate $\Sigma_{2}$ and $\Sigma_{3}$, and the proposition follows.

5. Proofs of Theorem 1 and Corollary. Take $y=x^{127 /(200 k)}$ in Proposition 1. Then the error term is $O\left(x^{173 /(200 k)+\varepsilon}\right)$. It remains to estimate the sums

$$
S_{1}^{ \pm}(y)=\sum_{d \leq y} \mu(d) \Delta_{k 1}^{ \pm}\left(x / d^{k}\right), \quad S_{2}^{ \pm}(y)=\sum_{d \leq y} \mu(d) \Delta_{k 2}^{ \pm}\left(x / d^{k}\right) .
$$

To estimate $S_{2}^{ \pm}(y)$, we need the estimate

$$
\Delta_{k 2}^{ \pm}(x) \ll x^{46 /(73 k)+\varepsilon},
$$

which is a consequence of the celebrated work of Huxley [3]. See also Nowak [13]. From this estimate we have

$$
S_{2}^{ \pm}(y) \ll x^{46 /(73 k)+\varepsilon} y^{27 / 73} \ll x^{173 /(200 k)+\varepsilon} .
$$

Now we estimate $S_{1}^{ \pm}(y)$. We only need to estimate

$$
S(D)=\sum_{d \sim D} \mu(d) \Delta_{k 1}^{ \pm}\left(x / d^{k}\right)
$$

for $1 \ll D \ll y$.

We suppose $(\kappa, \lambda)$ is an exponent pair such that $(3+\lambda) /(4(1+\kappa))<$ $1-1 / k$. If $D \ll x^{2 \kappa /((1+4 \kappa-\lambda) k)}$, then by the estimate

$$
\Delta_{k 1}^{ \pm}(u) \ll u^{1 / k-1 / k^{2}}
$$

we have

$$
S(D) \ll x^{\frac{1}{k}-\frac{1+2 \kappa-\lambda}{1+4 \kappa-\lambda} \cdot \frac{1}{k^{2}}} .
$$

Now suppose $D \gg x^{2 \kappa /((1+4 \kappa-\lambda) k)}$. By the expression of $\Delta_{k 1}^{ \pm}(u)$ we get

$$
S(D) \ll \frac{x^{1 / k-1 / k^{2}}}{D^{1-1 / k}}\left\{\sum_{l \leq U} l^{-1-1 / k}\left|S\left(l x^{1 / k}, D\right)\right|+D U^{-1 / k}\right\}
$$

for any $U>1$, where $S(W, D)$ is defined in the last section. We use Proposition 2 with $\alpha=2 / 5$ to bound $S\left(l x^{1 / k}, D\right)$ and take $U=D^{(1+4 \kappa-\lambda) /(2 \kappa)} x^{-1 / k}$ to get

$$
S(D) \ll x^{\frac{1}{k}-\frac{1+2 \kappa-\lambda}{1+4 \kappa-\lambda} \cdot \frac{1}{k^{2}}}+x^{\frac{327}{400 k}-\frac{73}{200 k^{2}}} \ll x^{\frac{1}{k}-\frac{1+2 \kappa-\lambda}{1+4 \kappa-\lambda} \cdot \frac{1}{k^{2}}}+x^{\frac{173}{200 k}} .
$$

This completes the proof of Theorem 1 . 
The Corollary follows from Theorem 1 if we take $(\kappa, \lambda)=(1 / 2,1 / 2)$ for $k=4,(\kappa, \lambda)=(19 / 126,86 / 126)$ for $k=5,(\kappa, \lambda)=(3 / 26,112 / 156)$ for $k=6,(\kappa, \lambda)=(2 / 18,13 / 18)$ for $k \geq 7$.

6. Proof of Theorem 2. Take $y=x^{1 / 6}$ in Proposition 1 . Then the error term is $O\left(x^{5 / 24+\varepsilon}\right)$. It suffices to estimate the sums

$$
S_{1}(D)=\sum_{d \sim D} \mu(d) \Delta_{41}^{+}\left(x / d^{4}\right), \quad S_{2}(D)=\sum_{d \sim D} \mu(d) \Delta_{42}^{+}\left(x / d^{4}\right)
$$

for any fixed $1 \ll D \ll y$.

$S_{1}(D)$ can be estimated in the same way as in the last section. Using Proposition 2 to bound $S\left(l x^{1 / 4}, D\right)$ by taking $(\kappa, \lambda)=(11 / 53,33 / 53)=$ $B A B A^{2} B A^{2} B(0,1)$ and $\alpha=2 / 5$, we get

$$
S_{1}(D) \ll x^{107 / 512} \log ^{5} x .
$$

Now we estimate $S_{2}(D)$. Without loss of generality, suppose $D \gg x^{1 / 12}$; otherwise by the trivial estimate

$$
S_{2}(D) \ll x^{3 / 16} D^{1 / 4} \ll x^{5 / 24} .
$$

For each fixed $d$, we write

$$
\begin{aligned}
& \sum_{x / 2<n^{4} d^{4} \leq x} \psi\left(\left(x / d^{4}-n^{4}\right)^{1 / 4}\right) \\
& =\sum^{1} \sum^{2} \psi\left(\left(x / d^{4}-n^{4}\right)^{1 / 4}\right)+O\left(x^{1 / 8} D^{-1 / 2}\right),
\end{aligned}
$$

where

$$
\begin{aligned}
& \mathrm{SC}\left(\sum^{1}\right): 1 \leq v \leq \frac{\log \left(x / D^{4}\right)}{8 \log 2}, \\
& \mathrm{SC}\left(\sum^{2}\right): \frac{x}{d^{4}}\left(1-2^{-v}\right)<n^{4} \leq \frac{x}{d^{4}}\left(1-2^{-v-1}\right) .
\end{aligned}
$$

Take $J=\max \left(x^{1 / 24} / 2^{v}, \log x\right)$, then by Lemma 4

$$
\begin{aligned}
\sum^{2} \psi\left(\left(x / d^{4}-n^{4}\right)^{1 / 4}\right)= & -2 i \operatorname{Re} \sum_{1 \leq h \leq J} \frac{1}{2 \pi h} \sum^{2} e\left(-h\left(x / d^{4}-n^{4}\right)^{1 / 4}\right) \\
& +O\left(\sum_{|h| \leq J} b(h) \sum^{2} e\left(-h\left(x / d^{4}-n^{4}\right)^{1 / 4}\right)\right)
\end{aligned}
$$

We first consider the sum

$$
S_{2}(d, h, v)=\sum^{2} e\left(-h\left(x / d^{4}-n^{4}\right)^{1 / 4}\right) .
$$


Similarly to formula (2.6) of Nowak [14], we can get

$$
\begin{aligned}
& S_{2}(d, h, v) \\
& \quad=e^{\pi i / 4} \sum^{3} g(r, d) e(F(r, d))+O\left(\frac{1}{\sqrt{h}} \cdot \frac{x^{1 / 8}}{D^{1 / 2}} 2^{-7 v / 8}+\log x\right),
\end{aligned}
$$

where

$$
\begin{aligned}
& F(r, d)=-\frac{x^{1 / 4}}{d}\left(h^{4 / 3}+r^{4 / 3}\right)^{3 / 4} \sim-\frac{r x^{1 / 4}}{d}, \\
& g(r, d)=\frac{h}{\sqrt{3}}(h r)^{-1 / 3} x^{1 / 8} d^{-1 / 2}\left(h^{4 / 3}+r^{4 / 3}\right)^{-5 / 8} \sim h^{2 / 3} r^{-7 / 6} x^{1 / 8} d^{-1 / 2}, \\
& \mathrm{SC}\left(\sum^{3}\right): h\left(2^{v}-1\right)^{3 / 4}<r \leq h\left(2^{v+1}-1\right)^{3 / 4} .
\end{aligned}
$$

Inserting (6.4) into (6.3) we have

$$
\begin{aligned}
\sum^{2} \psi\left(\left(x / d^{4}-n^{4}\right)^{1 / 4}\right)= & c \operatorname{Re} \sum_{1 \leq h \leq J} \frac{1}{2 \pi h} \sum^{3} g(r, d) e(F(r, d)) \\
& +O\left(\sum_{1 \leq h \leq J} b(h) \sum^{3} g(r, d) e(F(r, d))\right) \\
& +O\left(\log ^{2} x+\frac{x^{1 / 8}}{2^{7 v / 8} D^{1 / 2}}\right) .
\end{aligned}
$$

Thus

$$
\begin{aligned}
S_{2}(D)= & \sum_{d \sim D} \mu(d)\left(-8 \sum_{x / 2<n^{4} d^{4} \leq x} \psi\left(\left(x / d^{4}-n^{4}\right)^{1 / 4}\right)+O(1)\right) \\
= & -8 \sum_{d \sim D} \mu(d) \sum^{1} \sum^{2} \psi\left(\left(x / d^{4}-n^{4}\right)^{1 / 4}\right)+O\left(x^{1 / 8} D^{1 / 2}+D\right) \\
= & c \sum^{1} \sum_{1 \leq h \leq J} \frac{1}{h} \sum^{3} \sum_{d \sim D} \mu(d) g(r, d) e(F(r, d)) \\
& +O\left(\sum_{1 \leq h \leq J}^{1} \sum_{J} \frac{1}{J} \sum^{3}\left|\sum_{d \sim D} g(r, d) e(F(r, d))\right|\right)+O\left(x^{5 / 24}\right) \\
= & \sum^{4}+\sum^{5}+O\left(x^{5 / 24}\right), \text { say. }
\end{aligned}
$$

Using the exponent pair $(1 / 6,4 / 6)$ to estimate the sum over $d$ we easily find that

$$
\sum^{5} \ll x^{13 / 72} \log ^{2} x
$$

if we recall $D \gg x^{1 / 12}$.

We shall use Proposition 2 to estimate the sum over $d$ in $\sum^{4}$. Let

$$
W=x^{1 / 4}\left(h^{4 / 3}+r^{4 / 3}\right)^{3 / 4} \text {. }
$$


Take $(\kappa, \lambda)=(1 / 2,1 / 2)$ and $\alpha=2 / 5$ in Proposition 2 to get

$$
\begin{aligned}
\sum^{4} & \ll \sum^{1} \sum_{h} \frac{1}{h} \sum^{3} h^{2 / 3} r^{-7 / 6} \frac{x^{1 / 8+\varepsilon}}{D^{1 / 2}} \\
& \times\left(D^{4 / 5}+x^{1 / 24} r^{1 / 6} D^{7 / 12}+D^{3 / 2} x^{-1 / 8} r^{-1 / 4}\right) \\
& \ll x^{5 / 24+\varepsilon} .
\end{aligned}
$$

Combining (6.6)-(6.8) gives

$$
S_{2}(D) \ll x^{5 / 24+\varepsilon} .
$$

Now Theorem 2 follows from (6.1) and (6.9).

Acknowledgements. The author deeply thanks the referee for helpful suggestions.

\section{References}

[1] R. C. Baker, The square-free divisor problem, Quart. J. Math. Oxford Ser. 45 (1994), 269-277.

[2] E. Fouvry and H. Iwaniec, Exponential sums for monomials, J. Number Theory 33 (1989), 311-333.

[3] M. N. Huxley, Exponential sums and lattice points II, Proc. London Math. Soc. 66 (1993), 279-301.

[4] A. Ivić, The Riemann Zeta-function, Wiley, 1985.

[5] E. Krätzel, Lattice Points, Deutsch. Verlag Wiss., Berlin, 1988.

[6] - , Primitive lattice points in special plane domains and a related three-dimensional lattice point problem I, Forschungsergebnisse, FSU, Jena, N/87/11, 1987.

[7] H. L. Montgomery and R. C. Vaughan, The distribution of squarefree numbers, in: Recent Progress in Analytic Number Theory (Durham, 1979), Vol. 1, Academic Press, London, 1981, 247-256.

[8] B. Z. Moroz, On the number of primitive lattice points in plain domains, Monatsh. Math. 99 (1985), 37-43.

[9] W. Müller and W. G. Nowak, Lattice points in planar domains: applications of Huxley's "discrete Hardy-Littlewood method", in: Number-Theoretic Analysis (Vienna, 1988-89), Lecture Notes in Math. 1452, Springer, 1990, 139-164.

[10] - - - On a mean-value theorem concerning differences of two $k$-th powers, Tsukuba J. Math. 13 (1989), 23-29.

[11] W. G. Nowak, On sums of two coprime k-th powers, Monatsh. Math. 108 (1989), $47-57$.

[12] -, On sums and differences of two relative prime cubes, Analysis 15 (1995), 325341.

[13] - Primitive lattice points in starlike planar sets, Pacific J. Math. 170 (1997), $163-178$

[14] - On sums of two k-th powers: a mean-square bound for the error term, Analysis 16 (1996), 297-304. 
[15] W. G. Nowak, On sums and differences of two relative prime cubes II, Tatra Mt. Math. Publ. 11 (1997) (Proc. Czech and Slovak Number Theory Conference, 1995), 23-34.

[16] - On differences of two $k$-th powers of integers, Ramanujan J. 2 (1998), 421-440.

[17] J. D. Vaaler, Some extremal problems in Fourier analysis, Bull. Amer. Math. Soc. 12 (1985), 183-216.

Department of Mathematics

Shandong Normal University

Jinan, 250014, Shandong

P.R. China

E-mail: wgzhai@jn-public.sd.cninfo.net

Received on 30.1.1998

and in revised form on 11.9.1998 